Classification

Physics Abstracts

$32.80 \mathrm{~B}-42.55 \mathrm{H}-42.60 \mathrm{~F}$

\title{
Résonance magnétique dans un laser à gaz : observation à l'aide de la modulation haute fréquence du faisceau laser
}

\section{J. Hamel}

Université de Caen, Laboratoire de Spectroscopie Atomique, associé au C.N.R.S., 14032 Caen Cedex, France

\author{
et J. P. Barrat \\ Université de Caen, U.E.R. de Sciences, 14032 Caen Cedex, France
}

(Reçu le 13 juillet 1983, révisé le 7 octobre, accepté le 19 octobre 1983)

\begin{abstract}
Résumé. - Dans un laser à $\mathrm{He}-\mathrm{Cd}^{+}$, l'émission laser polarisée crée une inégalité de population entre les sous-niveaux Zeeman du niveau supérieur ${ }^{2} D_{5 / 2}$ de la transition laser. Lors d'une résonance magnétique dans ce niveau ${ }^{2} D_{5 / 2}$, le faisceau laser est modulé à la fréquence du champ H.F. ou à une fréquence harmonique.

Abstract. - In a He-Cd ${ }^{+}$laser, a population inequality between the Zeeman sublevels of the upper level ${ }^{2} \mathrm{D}_{5 / 2}$ of the laser transition is created by the polarized laser beam itself. If the $\mathrm{Cd}^{+}$ions in the ${ }^{2} \mathrm{D}_{5 / 2}$ level are submitted to magnetic resonance, the laser beam is modulated at the R.F. field frequency or at a harmonic frequency.
\end{abstract}

\section{Introduction.}

Les lasers ont souvent été utilisés comme sources lumineuses pour procéder à des expériences de résonance magnétique dans des états atomiques excités, mais il est rare que l'expérience ait porté sur les atomes eux-mêmes responsables de l'émission laser; dans ce dernier cas [1-5] la résonance magnétique était détectée par la variation de la polarisation de la lumière émise et le rapport signal sur bruit était généralement médiocre. Nous présentons ici quelques expériences de résonance magnétique sur les atomes constituant le milieu amplificateur d'un laser à gaz, dans lesquelles nous détectons la résonance magnétique par la modulation H.F. de la lumière émise par le laser, à la fréquence du champ de radio-fréquence ou à une fréquence harmonique. Ces modulations, bien connues [6,7] dans le cas d'une vapeur atomique optiquement alignée (ou orientée) soumise à une résonance magnétique, n'avaient, à notre connaissance, encore jamais été étudiées directement sur un laser. Outre l'intérêt de l'étude fondamentale de ces phénomènes, cette méthode constitue un procédé de modulation H.F. d'un laser facile à mettre en œuvre et relativement peu coûteux. 


\section{Principe et description de l'expérience.}

La plupart de nos expériences ont porté sur la raie $4416 \AA$ du spectre de CdII $\left[5^{2} \mathrm{P}_{3 / 2}-\right.$ $\left.\left(4 d^{9} 5 s^{2}\right)^{2} D_{5 / 2}\right]$ émise par un laser $\mathrm{He}-\mathrm{Cd}^{+}[8]$. Les durées de vie des niveaux mis en jeu sont de l'ordre de $700 \mathrm{~ns}$ pour le niveau supérieur et de $2,7 \mathrm{~ns}$ pour le niveau inférieur $[9,10]$. Les lasers utilisés ont été construits comme indiqué dans les références 11,12 et 13 . La cavité est constituée de miroirs multidiélectriques sphériques disposés au voisinage de la position confocale. Leurs rayons de courbure sont de $1 \mathrm{~m}$. Avec un miroir de sortie de coefficient de réflexion égal à $98 \%$, la puissance émise par nos lasers ne dépasse pas une dizaine de milliwatts. La direction du vecteur électrique e de l'onde lumineuse est imposée par les fenêtres à incidence brewstérienne du tube laser. Ce tube est placé dans un champ magnétique statique $\mathbf{B}_{0}$ et dans un champ R.F. $\mathbf{B}_{1}$ perpendiculaire à $\mathbf{B}_{0}$. Le champ $\mathbf{B}_{0}$ est obtenu à l'aide d'une paire de bobines rectangulaires de 200 spires chacune et de dimension $0,80 \times 0,12 \times 0,05 \mathrm{~m}$. Elles sont placées de part et d'autre du tube en " position d'Helmholtz ». Le champ R.F. est créé par deux spires rectangulaires disposées de chaque côté du tube et constituant la self d'un circuit accordé à la fréquence $N$ du champ oscillant $\mathbf{B}_{1}$. Pour les fréquences supérieures à $100 \mathrm{MHz}$ nous adoptons une configuration de fils de Lecher [2]. La figure 1 montre le dispositif expérimental.

Les sous-niveaux Zeeman du niveau ${ }^{2} D_{5 / 2}$ sont peuplés par collision de Penning de manière isotrope, mais l'émission laser étant polarisée, ils ne se dépeuplent pas à la même vitesse. A l'état stationnaire et en l'absence de résonance magnétique, il en résulte une inégalité de population entre ces sous-niveaux Zeeman [14-16].

Lorsqu'on soumet les ions $\mathrm{Cd}^{+}$excités dans le niveau ${ }^{2} \mathrm{D}_{5 / 2}$ au champ de radio-fréquence $\mathbf{B}_{1}$, les éléments non-diagonaux de la matrice densité représentant ces ions sont modulés à la fréquence $N$ et aux fréquences harmoniques $[6,7]$. Il en est donc de même de la lumière émise en émission induite. Le taux de modulation n'est important qu'au voisinage de la résonance magnétique et sa variation avec le champ statique $B_{0}$ (pour une valeur de $N$ donnée) permet d'observer cette résonance. On notera que l'inégalité de population entre sous-niveaux Zeeman est provoquée par l'émission induite due au faisceau laser lui-même; or la résonance magnétique produit une modulation de l'intensité de ce faisceau. On pourrait donc s'attendre à des effets non linéaires : inégalité de population " initiale " modulée, modification en conséquence de la valeur du taux de modulation pour des champs $B_{0}$ et $B_{1}$ donnés, etc... En fait le taux de modulation obtenu pour l'intensité du faisceau est assez faible, de sorte que de tels effets peuvent être négligés dans nos expériences.

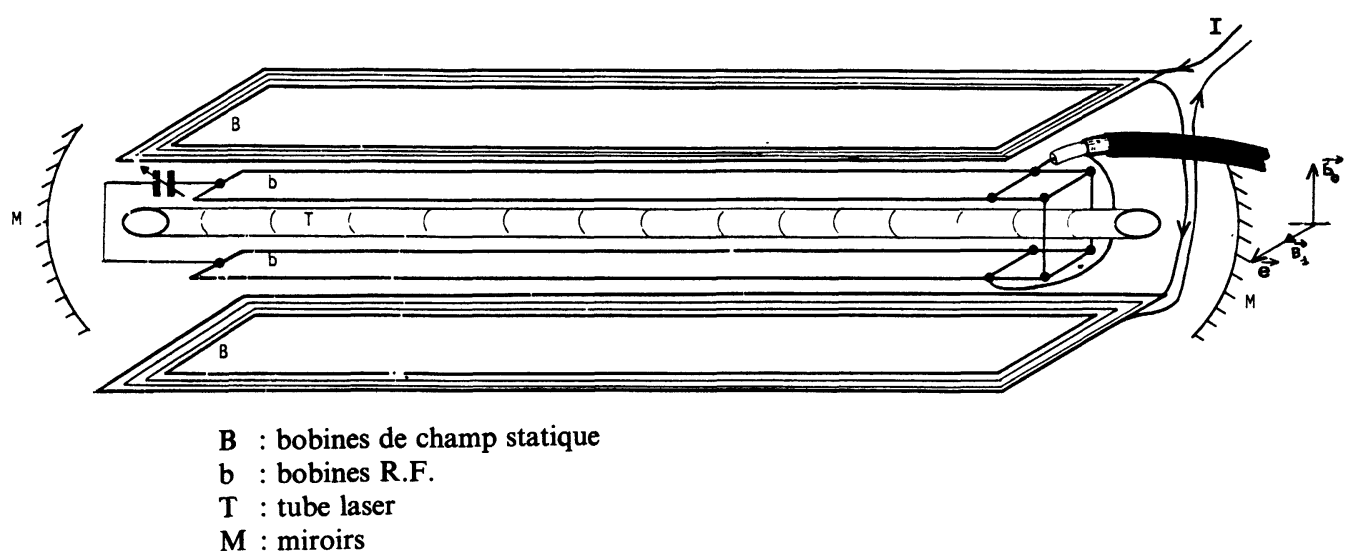

Fig. 1. - Dispositif expérimental.

[Experimental apparatus.] 


\section{Résultats déjà obtenus.}

Lorsque le champ $\mathbf{B}_{0}$ est perpendiculaire à la direction du vecteur $\mathbf{e}$ (polarisation $\sigma_{+}+\sigma_{-}$ cohérente) nous détectons une modulation à la fréquence $2 N$ double de celle du champ R.F. Le taux de modulation est de l'ordre de quelques pour-cent. Si l'on tourne la direction de $\mathbf{B}_{0}$ pour l'amener à $45^{\circ}$ du vecteur $\mathbf{e}$, (polarisation $\pi+\sigma$ cohérente) on observe aussi une modulation à la fréquence $N$. Ces effets sont parfaitement conformes à ce que donnent les prévisions théoriques bien connues dans ce genre d'expériences compte tenu des conditions de polarisation à l'excitation et à la détection. En outre une analyse de cette modulation H.F. à l'aide d'une détection de phase montre que les formes des courbes de résonance observées sont tout à fait semblables aux formes de raies obtenues dans les expériences habituelles de double résonance [6, 7]. Cet accord peut se justifier par le fait que le spectre du laser comporte de nombreux modes et aucune précaution particulière n'est prise pour les stabiliser. Dans ces conditions, on peut considérer que le faisceau laser a un spectre du type «broad line » [17]; ce faisceau joue dans l'expérience un double rôle : créer l'inégalité de population et la détecter. L'expérience est donc très analogue aux expériences traditionnelles de pompage optique dans un état fondamental, l'absorption étant remplacée par l'émission induite. La particularité est ici que les atomes soumis à la résonance magnétique sont aussi la source du faisceau qui joue ce double rôle.

D'autre part, la position et la largeur des résonances observées montrent bien que c'est la résonance du niveau supérieur ${ }^{2} \mathrm{D}_{5 / 2}$ de la transition laser que nous détectons. La faible durée de vie du niveau inférieur nécessiterait, en effet, des puissances R.F. beaucoup plus importantes que celles utilisées.

Dans la suite de cet article nous ne considérons que le cas où $\mathbf{B}_{0}$ et $\mathbf{e}$ sont perpendiculaires (modulation détectée à la fréquence $2 N$ ). Si l'on fait varier la fréquence $N$, le taux de modulation détecté lors de la résonance magnétique décroît lorsque $N$ augmente. Il devient difficile à mesurer quand $N$ dépasse $30 \mathrm{MHz}$, mais il varie de façon résonnante lorsque $2 N$ passe par la valeur $144 \mathrm{MHz}$ (équidistance des modes longitudinaux de la cavité laser). Pour les expériences aux fréquences $N$ faibles (inférieures à $30 \mathrm{MHz}$ ), il est avantageux d'appliquer la résonance magnétique sur toute la longueur du tube laser. Mais on ne peut plus observer ainsi de modulation à $144 \mathrm{MHz}(N=72 \mathrm{MHz})$. En effet les phases des différentes contributions à la modulation sur toute l'étendue de la cavité sont telles que le signal résultant est nul. Un calcul élémentaire, confirmé par l'expérience, montre que l'on obtient le signal optimal lorsque la résonance magnétique agit sur une moitié de la cavité, comprise entre son centre et un miroir.

A l'aide d'un analyseur de spectre (Hewlett-Packard 8554B + 8552B), on peut étudier le signal modulé reçu par une photodiode rapide. On maintient constant le champ $B_{0}$, ainsi que la grandeur et la fréquence $N=72 \mathrm{MHz}$ du champ $\mathbf{B}_{1}$. La courbe en trait plein de la figure 2 montre l'amplitude du signal lorsqu'on balaye la fréquence d'analyse de quelques dizaines de $\mathrm{kHz}$ de part et d'autre de $144 \mathrm{MHz}$; le taux de modulation maximal a une valeur voisine de $10 \%$. La courbe en pointillé montre le signal lorsqu'on "désaccorde » le champ statique $B_{0}$, $N$ et $B_{1}$ étant maintenus constants. Ce dernier signal, d'une largeur voisine de $25 \mathrm{kHz}$ est essentiellement dû aux battements entre les modes longitudinaux du laser. Il semble donc que, lorsqu'on accorde le champ $B_{0}$ sur la résonance magnétique du niveau ${ }^{2} \mathrm{D}_{5 / 2}$, les fluctuations de fréquence de ces battements diminuent au profit d'une seule composante intense à $144 \mathrm{MHz}$. Ceci peut être rapproché des effets de blocage de modes induits par une modulation intra-cavité à l'aide de dispositif acousto-optique [18]. Néanmoins, ni l'étude de la variation temporelle du signal de sortie (observée à l'aide d'un oscilloscope large bande) ni l'analyse fréquentielle (harmonique) ne permettent d'affirmer l'établissement d'un couplage caractérisé entre les modes longitudinaux.

La figure 3 montre l'allure du signal de résonance magnétique observé à $144 \mathrm{MHz}$. La fréquence $N=72 \mathrm{MHz}$ et la grandeur du champ $\mathbf{B}_{1}$ sont maintenues constantes, ainsi que la fréquence d'analyse fixée à $2 N=144 \mathrm{MHz}$. La bande passante de l'analyseur de spectre est 


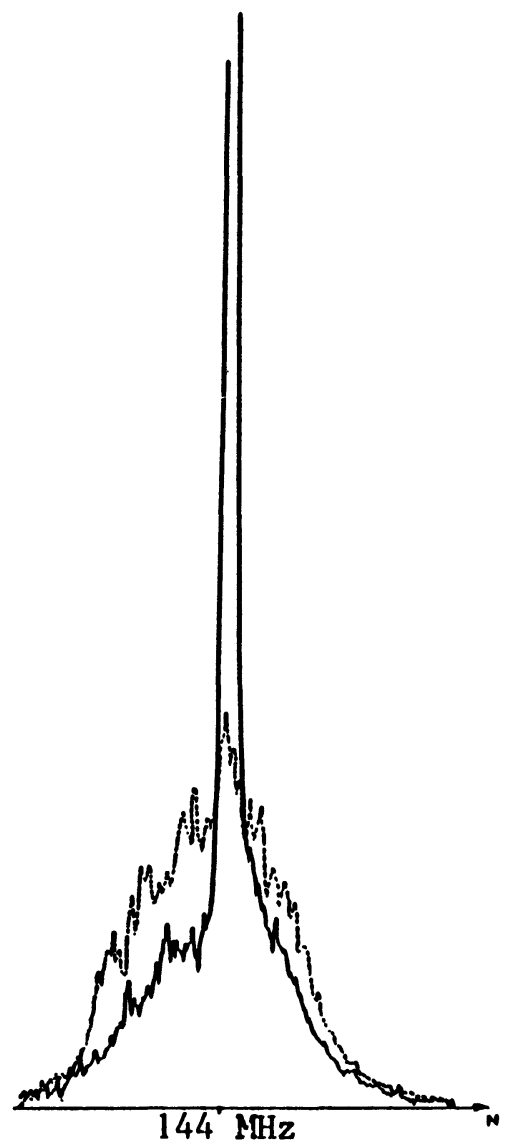

$10 \mathrm{kHz}$

Fig. 2. - Spectre de la modulation du faisceau émis par le laser au voisinage de $144 \mathrm{MHz}$.

[Spectrum of the modulation of the laser beam in the vicinity of $144 \mathrm{MHz}$.]

$1 \mathrm{kHz}$ et la constante de temps de la chaîne de mesure après détection est $0,1 \mathrm{~s}$. On distingue nettement les résonances des isotopes impairs présents dans la vapeur de cadmium. D'autres expériences ont été réalisées à une fréquence $2 N$ voisine de $288 \mathrm{MHz}$. Elles nous ont permis de détecter une autre variation résonnante de la modulation avec un rapport signal sur bruit voisin de 30 .

\section{Conclusion.}

Ces premiers résultats sur le laser $\mathrm{He}_{-} \mathrm{Cd}^{+}$montrent que l'observation de la modulation H.F. due à la résonance magnétique permet aisément la détection de cette résonance directement à l'intérieur d'un laser à gaz. Le bruit propre du laser a un spectre particulièrement intense aux fréquences inférieures à $1 \mathrm{MHz}$; ce type de détection modulée de la résonance magnétique permet donc un excellent rapport signal sur bruit. D'autres observations préliminaires ont été faites sur le laser $\mathrm{He}-\mathrm{Ne}$ (raies $6328 \AA, 1,15 \mu \mathrm{m}$ et 3,39 $\mu \mathrm{m}$ ). Malgré les durées de vie plus courtes des niveaux mis en jeu, nous détectons des signaux modulés avec un très bon rapport signal sur 


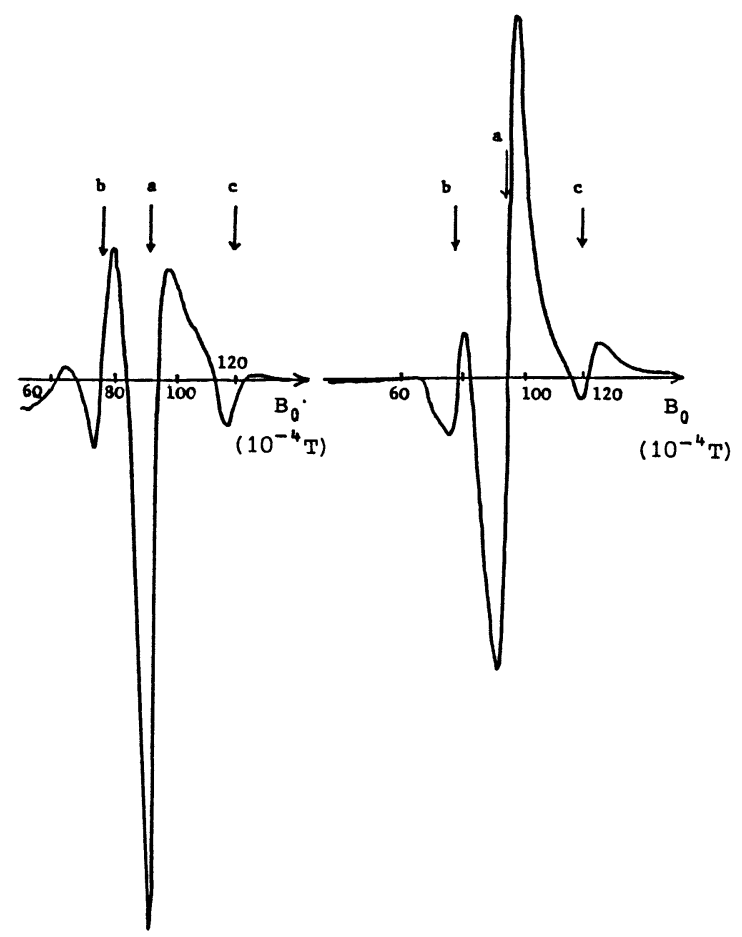

Fig. 3. - Signal de résonance magnétique à $144 \mathrm{MHz}$ (les 2 courbes correspondent à 2 réglages de phase en quadrature du dispositif de détection de la modulation du flux lumineux). Les flèches $a, b, c$ montrent les résonances aux facteurs de Landé $g=1,2$ (isotopes pairs) et $g=1,4$ et $g=1$ (isotopes impairs).

[Magnetic resonance signal at $144 \mathrm{MHz}$ (the 2 curves correspond to 2 different phase choices, in quadrature with each other, for the detection of the light intensity modulation). The arrows $a, b, c$ show the resonance at the Landé- $g$ factors $g=1.2$ (even isotopes) and $g=1.4$ and $g=1$ (odd isotopes).]

bruit. L'étude plus détaillée des phénomènes observés est en cours. D'autres résultats préliminaires très encourageants ont été aussi obtenus sur le laser He-Xe $(3,5 \mu \mathrm{m})$. Il semble évident que cette méthode peut se généraliser à de nombreux autres types de laser à gaz. La modulation du signal peut en outre constituer une application intéressante de techniques propres à la spectroscopie hertzienne, et faciles à mettre en œuvre.

\section{Remerciements.}

Les auteurs remercient l'ANVAR pour l'aide financière accordée à ce travail, dans le cadre d'un contrat de valorisation de la recherche. 


\section{Bibliographie}

[1] Carroll, T. O. et Wolga, G. J., IEEE J. Quant. Electronics QE 2 (1966) 456.

[2] YabuZaki, T. et Ogawa, T., J. Appl. Phys. 39 (1968) 4477.

[3] CARroll, T. O., IEEE J. Quant. Electronics QE 6 (1970) 516.

[4] Yabuzaki, T., Mita, N. et Ogawa, T., Phys. Rev. Lett. 29 (1972) 336.

[5] Yabuzaki, T. et Ogawa, T., J. Phys. Soc. Japan 34 (1973) 769.

[6] Dodd, J. N., Fox, W. N., Series, G. W. et Taylor, M. J., Proc. Phys. Soc. 74 (1959) 789 ; Dodd, J. N. et Series, G. W., Proc. R. Soc. A 263 (1961) 359 ; Kibble, B. P. et Series, G. W., Proc. R. Soc. A 274 (1963) 213.

[7] Barrat, J. P., Proc. R. Soc. A 263 (1961) 371.

[8] Collins, G. J., J. Appl. Phys. 44 (1973) 4633.

[9] Barrat, M. et Barrat, J. P., C.R. Hebd. Séan. Acad. Sci. 257 (1963) 1463 ; Barrat, M., Thèse Caen 1965.

[10] Hamel, J. et Barrat, J. P., Opt. Commun. 10 (1974) 331 ; Hamel, J., Thèse Caen 1977.

[11] Fendley, J. R., Gorog, I., Hernqvist, K. G. et Sun, C., R.C.A. Rev. 30 (1969) 422.

[12] Goldsborough, J. P., Appl. Phys. Lett. 15 (1969) 159.

[13] Silfyast, W. T. et Szeto, L. M., Appl. Phys. Lett. 19 (1971) 445.

[14] Decomps, B. et Dumont, M., C.R. Hebd. Séan. Acad. Sci. 262B (1966) 1004 ; Decomps, B. et Dumont, M., J. Physique 29 (1968) 181.

[15] Boyarskij, K. K., Kobzar, O. M. et Kotlikov, E. N., Opt. Spectrosc. 41 (1976) 96.

[16] Polishchuk, V. A., Todorov, G., Khartung, K. et Ghaika, K. P., Opt. Spectrosc. 34 (1973) 709.

[17] Ducloy, M., Thèse Paris 1973; Ducloy, M., Phys. Rev. A 8 (1973) 1844.

[18] Silfvast, W. T. et Smith, P. W., Appl. Phys. Lett. 17 (1970) 70. 